\title{
Picometric Ballistic (non-Brownian) Thermal Movements in Photonic Nanostructures
}

\author{
T. Liu' ${ }^{1}$ J. Y. Ou ${ }^{1}$, K. F. Macdonald ${ }^{1}$, and N. I. Zheludev ${ }^{1,2}$ \\ 1. Optoelectronics Research Centre and Centre for Photonic Metamaterials, University of Southampton, Southampton, SO17 1BJ, UK \\ 2. Centre for Disruptive Photonic Technologies, TPI, SPMS, Nanyang Technological University, Singapore 637371 \\ Authore-mail address: tongjun.liu@soton.ac.uk
}

\begin{abstract}
Components of photonic nanostructures exhibit picometric thermal movements affecting their optical properties. We report the first observation of ballistic (non-Brownian) thermal motion of a microcantilever, at timescales shorter than those of the erratic Brownian regime.
\end{abstract}

(C) 2020 The Authors

Nanoscale objects move fast and oscillate at $\mathrm{kHz}$ to $\mathrm{MHz}$ frequencies, and such movements (thermal or externally driven) underpin the functionality of a rapidly growing range of micro/nano-opto-mechanical (meta)materials, devices, sensors and systems. We report here on the detection and quantitative mapping of picometre (sub-atomic) amplitude cantilever movements, revealing the short-timescale ballistic regime of thermal motion.

The component parts of micro/nanostructured materials and devices are perturbed by collisions with atoms of ambient gas and by phonons in the crystal lattice of the constituent materials. As long ago as 1905 Einstein realized that the commonly held picture of Brownian motion, characterized by erratic, discontinuous changes in speed and direction, must break down at short time and length scales [1] - objects must move ballistically with smooth position trajectory (Fig. 1a) and a Maxwell-Boltzmann distribution of instantaneous velocities. Understandably at the time, he concluded that this regime, in which velocity changes on $\leq \mu$ s time and $\leq$ nm length scales, would be unobservable in practice. These scales are now accessible but mechanical thermodynamics in photonic and electro-optic nanostructures (Fig. 1b), where vibrations can become strongly coupled and highly nonlinear, remain underexplored because there are no routinely available technologies for quantitative spatial mapping of fast nano/picoscale motion. We show that real-time spectral analysis of variations in secondary electron emission from moving objects interrogated with a focused electron beam provides for such measurement, with sub-atomic displacement sensitivity.

Figure 2a illustrates the mechanism by which scattering of an incident electron beam transduces displacement, most effectively at the sharp edges of an object. The SE current $I(\boldsymbol{r})$, where $\boldsymbol{r}$ is positional coordinate, is sensitive to
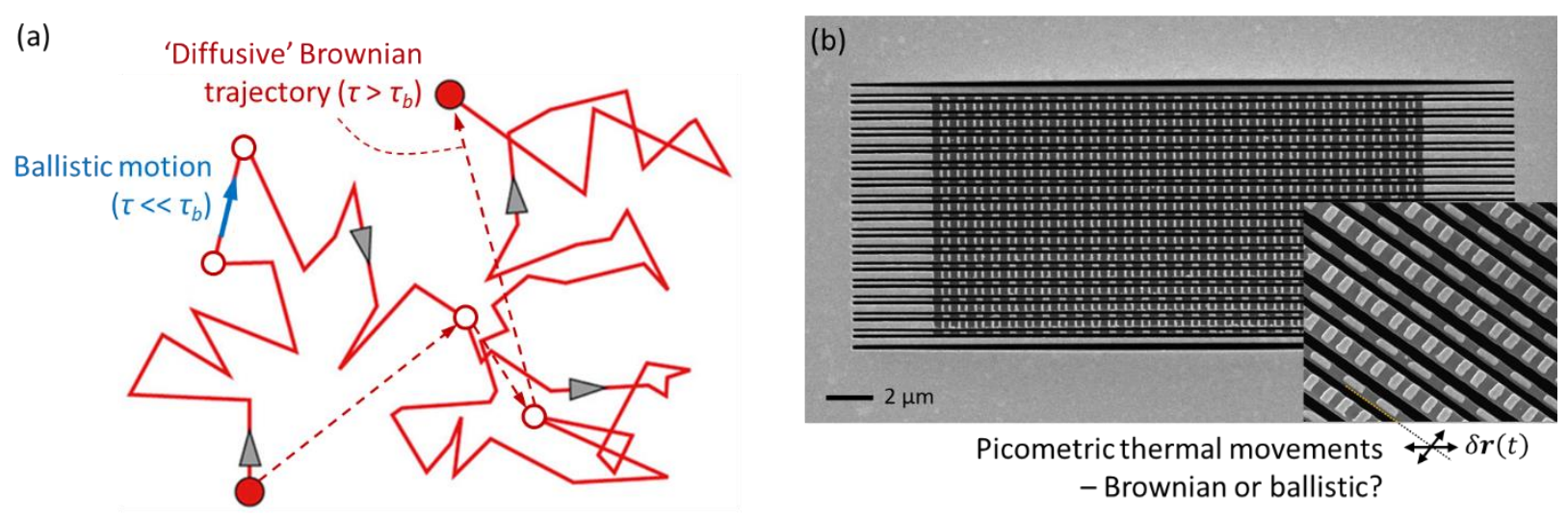

Figure 1. Diffusive vs. ballistic thermal motion. (a) Trajectory of a free particle undergoing thermal motion. What an observer sees depends upon the spatiotemporal resolution of measurements: An erratic 'Brownian' position trajectory is seen at timescales $\tau$ longer than the momentum relaxation time $\tau_{b}$; at much shorter time intervals ballistic motion is observed. (b) The component parts of nanomechanical photonic devices - here a gold/silicon nitride opto-mechanically reconfigurable metamaterial, are subject to thermal motion of characteristically pico- to nanometric amplitude. Can the ballistic regime be observed in such structures? 
small movements $\delta \boldsymbol{r}(t)$ of the object, with changes being proportional to the SE signal gradient in the displacement direction: $\delta I(\boldsymbol{r}, t) \sim(\nabla I(\boldsymbol{r}) \cdot \delta \boldsymbol{r}(t))$. With resolution ultimately constrained by the Poisson statistics of SE electrons impinging on the detector, a typical SEM is sensitive to displacements of sub-picometer amplitude - smaller by orders of magnitude than the $(\sim \mathrm{nm})$ electron beam spot size and the conventional static SE imaging resolution.

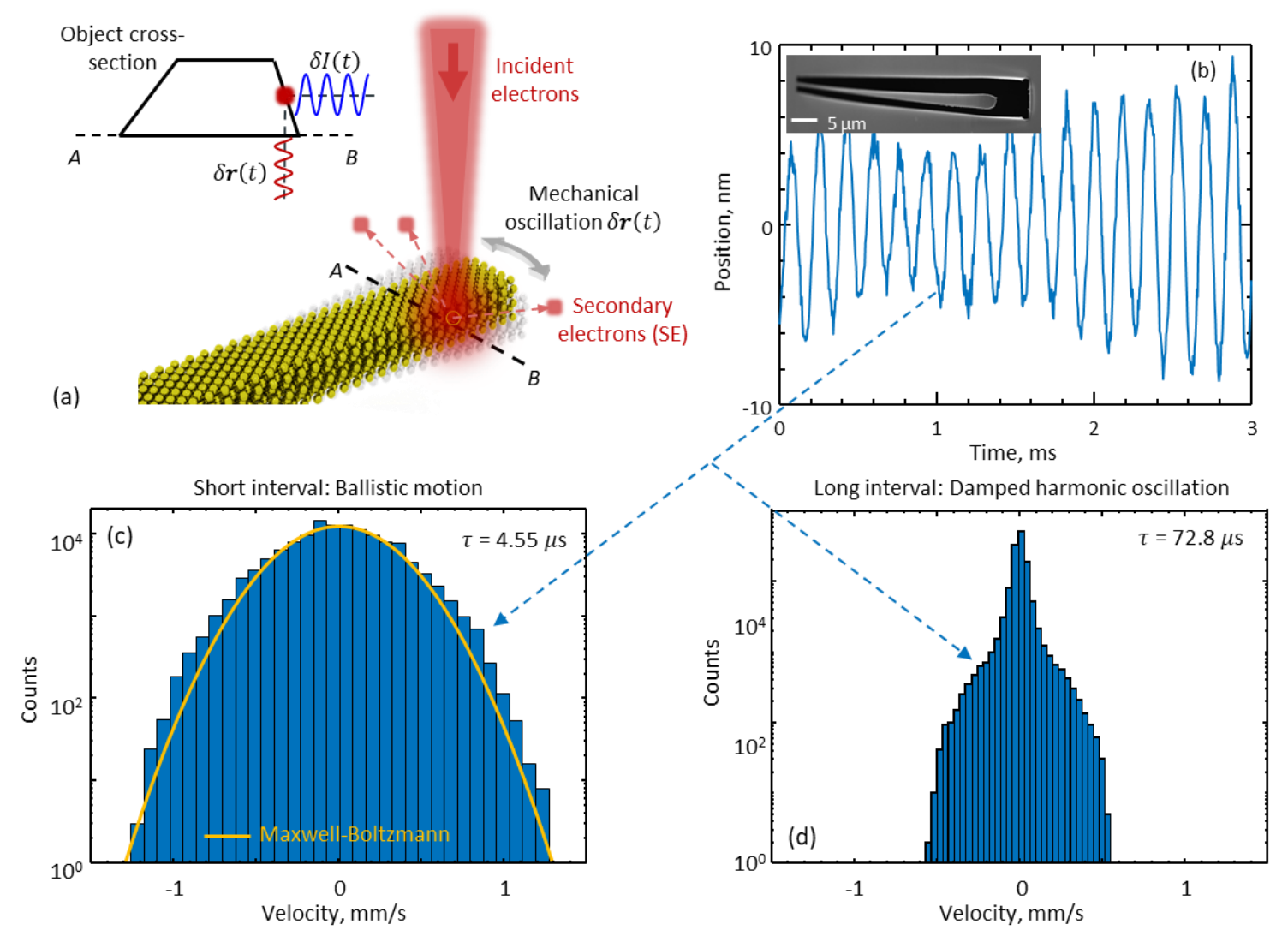

Figure 2. Measuring ballistic motion of a microcantilever. (a) Detecting pico-scale movement: when a beam of free electrons is focused at the edge of an oscillating nanostructure, the secondary electron current is modulated proportionally to the amplitude of movement and the gradient of the secondary electron emission at the injection point. (b) Real-time position trajectory of a free-standing, $30 \mathrm{~nm}$ thick gold cantilever [shown inset. (c, d) Corresponding velocity distributions at (c) short and (d) long time intervals.

Figure $2 \mathrm{~b}$ shows the measured real-time position trajectory of a microcantilever manufactured on free-standing $30 \mathrm{~nm}$ thick gold film. Its fundamental out-of-plane flexural mode has a period $T \sim 0.17 \mathrm{~ms}$. At short timescales $(\sim \mu \mathrm{s}$ $<T$ ) the distribution of instantaneous velocities (Fig. 2c) conforms to a Maxwell-Boltzmann distribution, as is expected in the ballistic regime. At longer intervals, greater than or equal to a significant fraction of $T$, the observed velocity distribution is that of a damped harmonic oscillator.

In summary, we are able to visualize real-time thermal movements occurring on at microsecond timescales with sub-atomic displacement sensitivity and nanoscale spatial resolution. This can enable quantitative measurement of the instantaneous velocity of micro/nanomechanical structures and spatial mapping of nanoscale oscillatory mode shapes and dynamic mechanical properties in a wide variety of nano-engineered objects, photonic and optoelectronic devices, and 2D material structures, while also presenting applications in the exploration of fundamental nonequilibrium statistical (opto)mechanics.

[1] A. Einstein, "Über die von der molekularkinetischen Theorie der Wärme geforderte Bewegung von in ruhenden Flüssigkeiten suspendierten Teilchen", Annalen der physik 322, 549-560 (1905). 